Portland State University

PDXScholar

Electrical and Computer Engineering Faculty

Publications and Presentations

$9-1-2009$

\title{
Stability Criteria for Spontaneously Pulsing Gas Lasers
}

Pitak Chenkosol

Portland State University

Lee W. Casperson

Portland State University

Follow this and additional works at: https://pdxscholar.library.pdx.edu/ece_fac

Part of the Electrical and Computer Engineering Commons

Let us know how access to this document benefits you.

Citation Details

Pitak Chenkosol and Lee W. Casperson, "Stability criteria for spontaneously pulsing gas lasers," J. Opt. Soc. Am. B 26, 939-945 (2009).

This Article is brought to you for free and open access. It has been accepted for inclusion in Electrical and Computer Engineering Faculty Publications and Presentations by an authorized administrator of PDXScholar. Please contact us if we can make this document more accessible: pdxscholar@pdx.edu. 


\title{
Stability criteria for spontaneously pulsing gas lasers
}

\author{
Pitak Chenkosol $^{1}$ and Lee W. Casperson ${ }^{2, *}$ \\ ${ }^{1}$ Department of Electrical and Computer Engineering, Portland State University, P. O. Box 751, \\ Portland, Oregon, 97207-0751 \\ ${ }^{2}$ Department of Electrical and Computer Engineering, and Center for Optoelectronics and Optical Communications, \\ University of North Carolina at Charlotte, 9201 University City Boulevard, \\ Charlotte, North Carolina, 28223-0001 \\ *Corresponding author: lcaspers@uncc.edu
}

Received January 9, 2009; revised March 3, 2009; accepted March 5, 2009;

posted March 5, 2009 (Doc. ID 105684); published April 6, 2009

Low-threshold spontaneous pulsations are known to occur in the output beams of certain high-gain gas lasers, and good agreement between the experimental observations and numerical models has been achieved. There have also been several analytical studies of threshold criteria for spontaneously pulsing lasers. However, the analytical studies are mostly not applicable to the lasers in which the pulsations have been observed. Stability criteria for high-gain gas lasers are derived in this study, and these criteria are compared with a previously modeled gas laser instability. (C) 2009 Optical Society of America

OCIS codes: $270.3430,270.3100,020.1670,140.3460$.

\section{INTRODUCTION}

Spontaneous pulsations in lasers have been of interest for many years, and this behavior has been studied experimentally and theoretically for lasers with various amplifying media and resonator configurations. In some of the most interesting cases, the pulsations arise directly from the basic semiclassical models with no added nonlinearities. The theoretical efforts have been both in the form of numerical solutions of the underlying equations and also as analytic studies of the corresponding perturbation stability criteria.

For the simplest homogeneously broadened lasers, there is at least the theoretical possibility of a basic onresonance spontaneous pulsation instability. However, the threshold for this instability is always at least nine times higher than the ordinary lasing threshold, which would usually be too high for rigorous experimental demonstration [1]. On the other hand, the corresponding instability in inhomogeneously broadened lasers can have a threshold that is very close to the lasing threshold. This effect has been studied many times since its discovery in Doppler-broadened xenon [1-5] and helium-neon [6-8] gas lasers, and it may be of practical value in applications requiring simple and efficient sources of periodic or chaotic optical pulsations. Thus, it would be useful to have as detailed an understanding as possible of the experimentally accessible laser instabilities.

Numerical studies of spontaneous pulsations in gas lasers have yielded good agreement with data from laboratory experiments, which gives confidence in the underlying models [2,9-11]. On the other hand, perturbation stability analyses have focused more on possible interesting or informative theoretical behavior without a particular emphasis on comparison with practical inhomogeneously broadened gas-laser systems. The Doppler
(Gaussian) variety of inhomogeneous broadening is especially important. For analytic tractibility some previous work has considered a hypothetical Lorentzian inhomogeneous broadening [12,13] and, in the inhomogeneously broadened limit, the exact form of the inhomogeneousbroadening function becomes unimportant [12]. The stability analysis should also allow practical values of the three basic decay rates (polarization, population, field). Some previous studies have, for simplicity, assumed that the polarization and population decay rates are either equal $[12,14]$ or differ by a factor of two [13], but these assumptions limit applicability for existing laser systems of interest. The objective of the present work has been to obtain stability criteria for basic Doppler-broadened ring lasers with arbitrary values for each of the decay rates and thus better compatibility with known or potential spontaneously pulsing lasers.

Previous studies of instability in ring lasers have shown that it is important to distinguish two types of stability criteria [15]. The first of these (type 1) concerns the smallest pump rate for which infinitesimal perturbations of the steady-state solution will increase with time. Commonly, the pump rate is represented by a threshold parameter, which normalizes the actual pump to the pump required for the laser to reach the ordinary lasing threshold. The second (type 2) concerns the smallest pump rate for which large amplitude pulsations will not damp away, and one finds that pulsations can sometimes be sustained at pumping levels well below the type 1 perturbation instability threshold. However, for practical spontaneously pulsing gas lasers, inhomogeneous broadening is dominant, and numerical calculations have shown that in this limit the two instability criteria become indistinguishable $[15,16]$. Thus, for analytical expedience only the type 1 instability criteria are considered here. 
The basic theoretical model for a uniform plane-wave in a ring-laser oscillator is established in Section 2. The steady-state solutions of this model are also derived. A perturbation stability analysis of the model is developed in Section 3. The results of this analysis are shown graphically in Section 4 and are applied to published data for spontaneously pulsing xenon lasers. The instability curves are consistent with the data and should be usable for predicting the possibility of spontaneous pulsations in other laser systems.

\section{MODEL}

The starting point for this analysis is a set of MaxwellSchrödinger equations that has been the basis for several previous studies of laser instabilities [9]. This general model permits a wide range of decay rates and resonator configurations. One important special case is the uniform plane-wave model for a Doppler broadened ring-laser oscillator in which the Doppler width is large compared to the homogeneous linewidth (the inhomogeneous limit). This model applies well to one of the best documented spontaneous-pulsation laser instabilities. As reduced to its normalized three-decay-rate form, this model can be written as in Eqs. (38)-(41) in [15]:

$$
\begin{aligned}
\frac{\partial P_{r}(V, t)}{\partial t}= & -\gamma\left[P_{r}(V, t)-V P_{i}(V, t)\right], \\
\frac{\partial P_{i}}{\partial t}(V, t)= & -\gamma\left\{P_{i}(V, t)+V P_{r}(V, t)+A(t)\right. \\
& \left.\times\left[D_{0}+D(V, t)\right]\right\}, \\
\frac{\partial D(V, t)}{\partial t}= & -\gamma_{d}\left[D(V, t)-A(t) P_{i}(V, t)\right], \\
\frac{\mathrm{d} A(t)}{\mathrm{d} t}= & -\gamma_{c}\left[A(t)+\int_{-\infty}^{\infty} P_{i}(V, t) \mathrm{d} V\right] .
\end{aligned}
$$

The independent variables in Eqs. (1)-(4) include the time $t$ and the normalized atomic or molecular velocity $V=k v / \gamma$ where $k$ is the propagation constant, $v$ is the actual velocity, and $\gamma$ is the polarization decay rate. The dependent variables in Eqs. (1)-(4) include the real part of the complex velocity- and time-dependent polarization $P_{r}(V, t)$ and the imaginary part of this polarization $P_{i}(V, t)$, the unsaturated population difference $D_{0}$, the saturation-induced correction to the population difference $D(V, t)$, and the electric field amplitude $A(t)$. The decay rates include the polarization decay rate $\gamma$, the population-difference decay rate $\gamma_{d}$, and the electric-field cavity decay rate $\gamma_{c}$.

Instability criteria for lasers are generally expressed in terms of the laser threshold parameter. This parameter expresses the actual pumping rate of a laser in terms of the minimum pumping required for the laser to reach the basic laser oscillation threshold. In the following paragraphs the threshold parameter is derived for the laser described by Eqs. (1)-(4).
Considering first the laser at steady state, i.e., $\partial / \partial t=0$, Eqs. (1)-(4) reduce to

$$
\begin{aligned}
& P_{r s}(V)=V P_{i s}(V), \\
& P_{i s}(V)=-V P_{r s}(V)-A_{s}\left(D_{0}+D_{s}(V)\right),
\end{aligned}
$$

$$
D_{s}(V)=A_{s} P_{i s}(V) \text {, }
$$

$$
A_{s}=-\int_{-\infty}^{\infty} P_{i s}(V) \mathrm{d} V
$$

Equations (5)-(7) can be combined to give

$$
P_{i s}(V)=-\frac{A_{s} D_{0}}{\left(1+V^{2}+A_{s}^{2}\right)}
$$

Equations (8) and (9) can then be combined to obtain

$$
1=D_{0} \int_{-\infty}^{\infty} \frac{\mathrm{d} V}{\left(1+V^{2}+A_{s}^{2}\right)}=2 D_{0} \int_{0}^{\infty} \frac{\mathrm{d} V}{\left(1+V^{2}+A_{s}^{2}\right)}=\frac{D_{0} \pi}{\sqrt{1+A_{s}^{2}}}
$$

It is convenient to define a threshold parameter $\mathfrak{R}$ that references the population difference to its value when lasing commences:

$$
\Re=\pi D_{0}=\sqrt{1+A_{s}^{2}} \text {. }
$$

Therefore, the steady-state electric field can be written

$$
A_{s}^{2}=\mathfrak{R}^{2}-1
$$

In terms of the threshold parameter $\Re$, one can write Eq. (2) as

$$
\frac{\partial P_{i}(V, t)}{\partial t}=-\gamma\left[P_{i}(V, t)+V P_{r}(V, t)+A(t)\left(\frac{\Re}{\pi}+D(V, t)\right)\right] .
$$

\section{STABILITY ANALYSIS}

To analyze the stability behavior of the system we assume solutions in the form

$$
\begin{gathered}
P_{r}(V, t)=P_{r s}(V)+P_{r}^{\prime}(V, t), \\
P_{i}(V, t)=P_{i s}(V)+P_{i}^{\prime}(V, t), \\
D(V, t)=D_{s}(V)+D^{\prime}(V, t), \\
A(t)=A_{s}+A^{\prime}(t),
\end{gathered}
$$


where the primed quantities represent perturbations that are assumed to be small and of the same approximate magnitudes relative to each other. With these substitutions, Eqs. (1), (3), (4), and (13) can be rewritten

$$
\begin{aligned}
\frac{\partial P_{r}^{\prime}(V, t)}{\partial t}= & -\gamma\left[\left(P_{r s}(V)+P_{r}^{\prime}(V, t)\right)-V\left(P_{i s}(V)+P_{i}^{\prime}(V, t)\right)\right] \\
= & -\gamma\left[P_{r}^{\prime}(V, t)-V P_{i}^{\prime}(V, t)\right] \\
\frac{\partial P_{i}^{\prime}(V, t)}{\partial t}= & -\gamma\left[\left(P_{i s}(V)+P_{i}^{\prime}(V, t)\right)+V\left(P_{r s}(V)+P_{r}^{\prime}(V, t)\right)\right. \\
& \left.+\left(A_{s}+A^{\prime}(t)\right)\left(\frac{\Re}{\pi}+D_{s}(V)+D^{\prime}(V, t)\right)\right] \\
\cong & -\gamma\left[P_{i}^{\prime}(V, t)+V P_{r}^{\prime}(V, t)+A_{s} D^{\prime}(V, t)\right. \\
& \left.+A^{\prime}(t)\left(\frac{\Re}{\pi}+D_{s}(V)\right)\right] \\
\frac{\partial D^{\prime}(V, t)}{\partial t}= & -\gamma_{d}\left[\left(D_{s}(V)+D^{\prime}(V, t)\right)-\left(A_{s}+A^{\prime}(t)\right)\left(P_{i s}(V)\right.\right. \\
& \left.\left.+P_{i}^{\prime}(V, t)\right)\right] \\
\cong & -\gamma_{d}\left[D^{\prime}(V, t)-\left(A_{s} P_{i}^{\prime}(V, t)+A^{\prime}(t) P_{i s}(V)\right)\right]
\end{aligned}
$$

$$
\begin{aligned}
\frac{\mathrm{d} A^{\prime}(t)}{\mathrm{d} t} & =-\gamma_{c}\left[\left(A_{s}+A^{\prime}(t)\right)+\int_{-\infty}^{\infty}\left(P_{i s}(V)+P_{i}^{\prime}(V, t)\right) \mathrm{d} V\right] \\
& =-\gamma_{c}\left[A^{\prime}(t)+\int_{-\infty}^{\infty} P_{i}^{\prime}(V, t) \mathrm{d} V\right]
\end{aligned}
$$

where some of the steady-state dependent variables have been cancelled using Eqs. (5)-(8) and the "approximately equals" signs indicate the dropping of second-order perturbation terms.

Next, we introduce the new parameters $\delta=\gamma / \gamma_{c}, \rho$ $=\gamma_{d} / \gamma$, and $t^{\prime}=\gamma_{c} t$, and then we rewrite Eqs. (18)-(21) as

$$
\begin{aligned}
\frac{\partial P_{r}^{\prime}\left(V, t^{\prime}\right)}{\partial t^{\prime}}= & -\delta\left[P_{r}^{\prime}\left(V, t^{\prime}\right)-V P_{i}^{\prime}\left(V, t^{\prime}\right)\right], \\
\frac{\partial P_{i}^{\prime}\left(V, t^{\prime}\right)}{\partial t^{\prime}}= & -\delta\left[P_{i}^{\prime}\left(V, t^{\prime}\right)+V P_{r}^{\prime}\left(V, t^{\prime}\right)\right. \\
& \left.+A_{s} D^{\prime}\left(V, t^{\prime}\right)+A^{\prime}\left(t^{\prime}\right)\left(\frac{\Re}{\pi}+D_{s}(V)\right)\right],
\end{aligned}
$$

$$
\begin{aligned}
\frac{\partial D^{\prime}\left(V, t^{\prime}\right)}{\partial t^{\prime}}= & -\delta \rho\left[D^{\prime}\left(V, t^{\prime}\right)-\left(A_{s} P_{i}^{\prime}\left(V, t^{\prime}\right)\right.\right. \\
& \left.\left.+A^{\prime}\left(t^{\prime}\right) P_{i s}(V)\right)\right], \\
\frac{\mathrm{d} A^{\prime}\left(t^{\prime}\right)}{\mathrm{d} t^{\prime}}= & -\left[A^{\prime}\left(t^{\prime}\right)+\int_{-\infty}^{\infty} P_{i}^{\prime}\left(V, t^{\prime}\right) \mathrm{d} V\right] .
\end{aligned}
$$

Due to the first-order nature of Eqs. (22)-(25), and in order to investigate the long-term behavior of the system, we assume solutions of Eqs. (22)-(25) in the form

$$
\begin{aligned}
P_{r}^{\prime}\left(V, t^{\prime}\right) & =P_{r}^{\prime}(V) e^{\lambda t^{\prime}}, \\
P_{i}^{\prime}\left(V, t^{\prime}\right) & =P_{i}^{\prime}(V) e^{\lambda t^{\prime}}, \\
D^{\prime}\left(V, t^{\prime}\right) & =D^{\prime}(V) e^{\lambda t^{\prime}}, \\
A^{\prime}\left(t^{\prime}\right) & =A^{\prime} e^{\lambda t^{\prime}},
\end{aligned}
$$

where $\lambda$ is the complex-valued rate constant. Substituting the solutions from Eqs. (26)-(29) into Eqs. (22)-(25) one obtains

$$
\begin{aligned}
\lambda P_{r}^{\prime}(V)= & -\delta\left[P_{r}^{\prime}(V)-V P_{i}^{\prime}(V)\right] \\
(\lambda+\delta) P_{r}^{\prime}(V)= & \delta V P_{i}^{\prime}(V), \\
\lambda P_{i}^{\prime}(V)= & -\delta\left[P_{i}^{\prime}(V)+V P_{r}^{\prime}(V)+A_{s} D^{\prime}(V)\right. \\
& \left.+A^{\prime}\left(\frac{\Re}{\pi}+D_{s}(V)\right)\right], \\
(\lambda+\delta) P_{i}^{\prime}(V)= & -\delta\left[V P_{r}^{\prime}(V)\right. \\
& \left.+A_{s} D^{\prime}(V)+A^{\prime}\left(\frac{\Re}{\pi}+D_{s}(V)\right)\right],
\end{aligned}
$$

$$
\lambda D^{\prime}(V)=-\delta \rho\left[D^{\prime}(V)-\left(A_{s} P_{i}^{\prime}(V)+A^{\prime} P_{i s}(V)\right)\right],
$$$$
(\lambda+\delta \rho) D^{\prime}(V)=\delta \rho\left[A_{s} P_{i}^{\prime}(V)+A^{\prime} P_{i s}(V)\right]
$$

$$
\lambda A^{\prime}=-\left[A^{\prime}+\int_{-\infty}^{\infty} P_{i}^{\prime}(V) \mathrm{d} V\right],
$$

$$
(\lambda+1) A^{\prime}=-\int_{-\infty}^{\infty} P_{i}^{\prime}(V) \mathrm{d} V .
$$

Substituting $P_{r}^{\prime}(V)=\delta V P_{i}^{\prime}(V) /(\lambda+\delta)$ from Eq. (30) into Eq. (31) yields

$$
\begin{aligned}
(\lambda+\delta) P_{i}^{\prime}(V)= & -\delta\left[V\left(\frac{\delta V}{\lambda+\delta}\right) P_{i}^{\prime}(V)+A_{s} D^{\prime}(V)\right. \\
& \left.+A^{\prime}\left(\frac{\Re}{\pi}+D_{s}(V)\right)\right], \\
\left(\lambda+\delta+\frac{\delta^{2} V^{2}}{\lambda+\delta}\right) P_{i}^{\prime}(V)= & -\delta\left[A_{s} D^{\prime}(V)+A^{\prime}\left(\frac{\Re}{\pi}+D_{s}(V)\right)\right] .
\end{aligned}
$$

Combining Eqs. (32) and (34) then leads to 


$$
\begin{gathered}
\left(\lambda+\delta+\frac{\delta^{2} V^{2}}{\lambda+\delta}\right) P_{i}^{\prime}(V)=-\delta\left[A _ { s } \left\{\frac { \delta \rho } { \lambda + \delta \rho } \left(A_{s} P_{i}^{\prime}(V)\right.\right.\right. \\
\left.\left.\left.+A^{\prime} P_{i s}(V)\right)\right\}+A^{\prime}\left(\frac{\Re}{\pi}+D_{s}(V)\right)\right] \\
\left(\lambda+\delta+\frac{\delta^{2} V^{2}}{\lambda+\delta}+\frac{\delta^{2} \rho A_{s}^{2}}{\lambda+\delta \rho}\right) P_{i}^{\prime}(V) \\
=\frac{-\delta^{2} \rho A_{s}}{(\lambda+\delta \rho)}\left[\frac{-A_{s}(\Re / \pi)}{1+V^{2}+A_{s}^{2}}\right] A^{\prime}-\delta\left[(\Re / \pi)-\frac{A_{s}^{2}(\Re / \pi)}{1+V^{2}+A_{s}^{2}}\right] A^{\prime} \\
=\frac{\delta^{2} \rho(\Re / \pi) A_{s}^{2} A^{\prime}}{(\lambda+\delta \rho)\left(1+V^{2}+A_{s}^{2}\right)}-\frac{\delta(\Re / \pi)\left(1+V^{2}\right) A^{\prime}}{\left(1+V^{2}+A_{s}^{2}\right)} \\
=-\left[\frac{\delta(\Re / \pi) A^{\prime}}{1+V^{2}+A_{s}^{2}}\right]\left[1+V^{2}-\frac{\delta \rho A_{s}^{2}}{\lambda+\delta \rho}\right], \\
\left.\quad P_{i}^{\prime}(V)=-\frac{\delta(\Re / \pi) A^{\prime}}{1+V^{2}+A_{s}^{2}}\right]\left[1+V^{2}-\frac{\delta \rho A_{s}^{2}}{\lambda+\delta \rho}\right] \\
\left(\lambda+\delta+\frac{\delta^{2} V^{2}}{\lambda+\delta}+\frac{\delta^{2} \rho A_{s}^{2}}{\lambda+\delta \rho}\right)
\end{gathered}
$$$$
=\delta\left(\frac{\Re}{\pi}\right) \int_{-\infty}^{\infty} \frac{(\lambda+\delta)}{\left[1+V^{2}+A_{s}^{2}\right]}
$$$$
\times \frac{\left[(\lambda+\delta \rho)\left(1+V^{2}\right)-\delta \rho A_{s}^{2}\right] \mathrm{d} V}{\left[(\lambda+\delta \rho)\left((\lambda+\delta)^{2}+\delta^{2} V^{2}\right)+(\lambda+\delta) \delta^{2} \rho A_{s}^{2}\right]} .
$$

Substituting $A_{s}^{2}=\mathfrak{R}^{2}-1$ from Eq. (12) into Eq. (40), one can write

$$
\begin{aligned}
(\lambda+1)= & \delta\left(\frac{\mathfrak{R}}{\pi}\right) \int_{-\infty}^{\infty} \frac{(\lambda+\delta)}{\left[V^{2}+R^{2}\right]} \\
& \times \frac{\left[(\lambda+\delta \rho)\left(1+V^{2}\right)-\delta \rho\left(\Re^{2}-1\right)\right] \mathrm{d} V}{\left[(\lambda+\delta \rho)\left((\lambda+\delta)^{2}+\delta^{2} V^{2}\right)+(\lambda+\delta) \delta^{2} \rho\left(\Re^{2}-1\right)\right]} \\
= & 2 \delta\left(\frac{\mathfrak{R}}{\pi}\right)(\lambda+\delta) \int_{-\infty}^{\infty} \frac{1}{\left(V^{2}+\mathfrak{R}^{2}\right)} \\
& \times \frac{\left[(\lambda+\delta \rho)\left(1+V^{2}\right)-\delta \rho\left(\Re^{2}-1\right)\right] \mathrm{d} V}{\left[(\lambda+\delta \rho)\left((\lambda+\delta)^{2}+\delta^{2} V^{2}\right)+(\lambda+\delta) \delta^{2} \rho\left(\Re^{2}-1\right)\right]} .
\end{aligned}
$$

(36) To facilitate the integrations we now introduce the following substitutions:

$$
\begin{aligned}
& A_{2}=(\lambda+\delta \rho), \\
& B_{2}=-\delta \rho\left(\Re^{2}-1\right), \\
& C_{2}=(\lambda+\delta)^{2}, \\
& D_{2}=\delta^{2}, \\
& E_{2}=(\lambda+\delta) \delta^{2} \rho\left(\Re^{2}-1\right), \\
& F_{2}=\frac{A_{2}+B_{2}}{A_{2}}, \\
& G_{2}=\frac{A_{2} C_{2}+E_{2}}{A_{2} D_{2}} .
\end{aligned}
$$

The integral in Eq. (41) can be rewritten and carried out as

$$
\begin{aligned}
\int_{0}^{\infty} \frac{1}{\left(V^{2}+\mathfrak{R}^{2}\right)} \frac{\left[A_{2}\left(1+V^{2}\right)+B_{2}\right]}{\left[A_{2}\left(C_{2}+D_{2} V^{2}\right)+E_{2}\right]} \mathrm{d} V & =\int_{0}^{\infty} \frac{1}{\left(V^{2}+\mathfrak{R}^{2}\right)} \frac{\left[\left(A_{2}+B_{2}\right)+A_{2} V^{2}\right]}{\left[\left(A_{2} C_{2}+E_{2}\right)+A_{2} D_{2} V^{2}\right]} \mathrm{d} V \\
& =\frac{1}{D_{2}} \int_{0}^{\infty} \frac{1}{\left(V^{2}+\mathfrak{R}^{2}\right)} \frac{\left[\left(\frac{A_{2}+B_{2}}{A_{2}}\right)+V^{2}\right]}{\left[\left(\frac{A_{2} C_{2}+E_{2}}{A_{2} D_{2}}\right)+V^{2}\right]} \mathrm{d} V \\
& =\frac{1}{D_{2}} \int_{0}^{\infty} \frac{\left(F_{2}+V^{2}\right)}{\left(V^{2}+\mathfrak{R}^{2}\right)\left(G_{2}+V^{2}\right)} \mathrm{d} V
\end{aligned}
$$




$$
\begin{aligned}
& =\frac{1}{D_{2}\left(\Re^{2}-G_{2}\right)}\left[\left(\Re^{2}-F_{2}\right) \int_{0}^{\infty} \frac{\mathrm{d} V}{V^{2}+\mathfrak{R}^{2}}+\left(F_{2}-G_{2}\right) \int_{0}^{\infty} \frac{\mathrm{d} V}{G_{2}+V^{2}}\right] \\
& =\frac{1}{D_{2}\left(\mathfrak{R}^{2}-G_{2}\right)}\left[\left(\mathfrak{R}^{2}-F_{2}\right)\left(\frac{\pi}{2 \Re}\right)+\left(F_{2}-G_{2}\right)\left(\frac{\pi}{2 \sqrt{G_{2}}}\right)\right] \\
& =\frac{(\pi / 2)}{D_{2}\left(\Re^{2}-G_{2}\right)}\left[\frac{\Re^{2}-F_{2}}{\Re}+\frac{F_{2}-G_{2}}{\sqrt{G_{2}}}\right] .
\end{aligned}
$$

Combining Eqs. (41) and (49) then leads to

$$
\begin{gathered}
(\lambda+1)-2 \delta\left(\frac{\mathfrak{R}}{\pi}\right)(\lambda+\delta) \frac{(\pi / 2)}{D_{2}\left(\mathfrak{R}^{2}-G_{2}\right)}\left[\frac{\mathfrak{R}^{2}-F_{2}}{\mathfrak{R}}+\frac{F_{2}-G_{2}}{\sqrt{G_{2}}}\right]=0, \\
(\lambda+1)-\frac{\delta \Re(\lambda+\delta)}{D_{2}\left(\mathfrak{R}^{2}-G_{2}\right)}\left[\frac{\mathfrak{R}^{2}-F_{2}}{\mathfrak{R}}+\frac{F_{2}-G_{2}}{\sqrt{G_{2}}}\right]=0 .
\end{gathered}
$$

If the variables in Eqs. (42)-(48) are now substituted into Eq. (50), one obtains

$$
\begin{aligned}
0= & (\lambda+1)-\frac{\delta \Re(\lambda+\delta)}{\delta^{2}\left(\mathfrak{R}^{2}-\frac{A_{2} C_{2}+E_{2}}{A_{2} D_{2}}\right)} \times\left[\frac{\mathfrak{R}^{2}-\frac{A_{2}+B_{2}}{A_{2}}}{\mathfrak{R}}+\frac{\frac{A_{2}+B_{2}}{A_{2}}-\frac{A_{2} C_{2}+E_{2}}{A_{2} D_{2}}}{\sqrt{\frac{A_{2} C_{2}+E_{2}}{A_{2} D_{2}}}}\right] \\
= & (\lambda+1)-\frac{\delta \Re(\lambda+\delta)}{\delta^{2}\left[\mathfrak{R}^{2}-\frac{(\lambda+\delta \rho)(\lambda+\delta)^{2}+(\lambda+\delta) \delta^{2} \rho\left(\Re^{2}-1\right)}{(\lambda+\delta \rho) \delta^{2}}\right]} \\
& \times\left[\frac{\frac{(\lambda+\delta \rho)-\delta \rho\left(\Re^{2}-1\right)}{(\lambda+\delta \rho)}-\frac{(\lambda+\delta \rho)(\lambda+\delta)^{2}+(\lambda+\delta) \delta^{2} \rho\left(\Re^{2}-1\right)}{(\lambda+\delta \rho) \delta^{2}}}{\sqrt{\frac{(\lambda+\delta \rho)(\lambda+\delta)^{2}+(\lambda+\delta) \delta^{2} \rho\left(\Re^{2}-1\right)}{(\lambda+\delta \rho) \delta^{2}}}}\right] .
\end{aligned}
$$

Equation (51) is the fundamental outcome for this stability analysis. The complex exponent $\lambda$ implied by Eq. (51) represents the behavior of a small perturbation of the laser away from a steady-state operating point. A specific application of this formula is considered below.

\section{RESULTS}

At the instability threshold, the real part of the complex rate constant $\lambda$ must be zero. With the real part of $\lambda$ set to zero, one can write $\lambda=i v$ where $v$ is a positive real number that represents an oscillation frequency of the small perturbation. With the substitution of $\lambda=i v$ into Eq. (51) one obtains

$$
\begin{aligned}
0= & (i v+1)-\frac{\delta \Re(i v+\delta)}{\delta^{2}\left[\mathfrak{R}^{2}-\frac{(i v+\delta \rho)(i v+\delta)^{2}+(i v+\delta) \delta^{2} \rho\left(\mathfrak{R}^{2}-1\right)}{(i v+\delta \rho) \delta^{2}}\right]} \\
& \times\left[\frac{\mathfrak{R}^{2}-\frac{i v+\delta \rho-\delta \rho\left(\mathfrak{R}^{2}-1\right)}{(i v+\delta \rho)}}{\mathfrak{R}}+\frac{\frac{(i v+\delta \rho)-\delta \rho\left(\mathfrak{R}^{2}-1\right)}{(i v+\delta \rho)}-\frac{(i v+\delta \rho)(i v+\delta)^{2}+(i v+\delta) \delta^{2} \rho\left(\mathfrak{R}^{2}-1\right)}{(i v+\delta \rho) \delta^{2}}}{\sqrt{\frac{(i v+\delta \rho)(i v+\delta)^{2}+(i v+\delta) \delta^{2} \rho\left(\mathfrak{R}^{2}-1\right)}{(i v+\delta \rho) \delta^{2}}}}\right]
\end{aligned}
$$


Both real and imaginary parts of Eq. (52) must be simultaneously satisfied at the instability boundary. The results of the calculations for different values of $\delta$ and $\rho$ are values for $\nu$ and $\Re$ at the onset boundary for spontaneous pulsations. A specific set of stability curves is shown in Fig. 1. These results are similar to those obtained by brute force numerical methods and shown in Fig. 5 of [15]. Each curve in Fig. 1 represents the stability boundary below which the laser operates in a stable region, where small perturbations of steady-state solutions decay with time. Above each curve small perturbations of the steady-state solutions increase with time, indicating that the laser is unstable with respect to spontaneous pulsations.

It is now possible to compare the stability curves in Fig. 1 with data for known spontaneously pulsing inhomogeneously broadened gas lasers. The xenon laser operating at a wavelength of $3.51 \mu \mathrm{m}$ was the first such system to be known, with spontaneous pulsations having been observed in 1969 and understood and modelled by 1978 [1]. Stability criteria specific to xenon lasers were also reported previously [17], in contrast to the more universal results presented here.

The first point to consider in applying Fig. 1 is the degree of inhomogeneous broadening. It has been found from numerical computations that if the inhomogeneous linewidth exceeds the homogeneous linewidth by a factor of at least about ten, it is sufficient to carry out any stability computations in the inhomogeneous limit [16]. In this circumstance also, the two types of stability criteria converge [16]. Approximate xenon laser parameters are reviewed in [9]. Specifically, the homogeneous linewidth is about $\Delta \nu_{h}=5.2 \mathrm{MHz}$, while the Doppler linewidth is about $\Delta \nu_{d}=110 \mathrm{MHz}$. Thus, the natural damping ratio is $\varepsilon$ $=\Delta \nu_{h}(\ln 2)^{1 / 2} / \Delta \nu_{d}=5.2(\ln 2)^{1 / 2} / 110=0.039$, and the laser may be considered to be inhomogeneously broadened. The curves in Fig. 1 are applicable and represent the merged type 1 and type 2 stability boundaries.

Also from [9] it can be argued that the polarization decay rate for the xenon laser used in early experiments was $\gamma=16.3 \times 10^{6} \mathrm{sec}^{-1}$, the population difference decay rate $\gamma_{d}=8.33 \times 10^{5} \mathrm{sec}^{-1}$, and the electric-field cavity de-

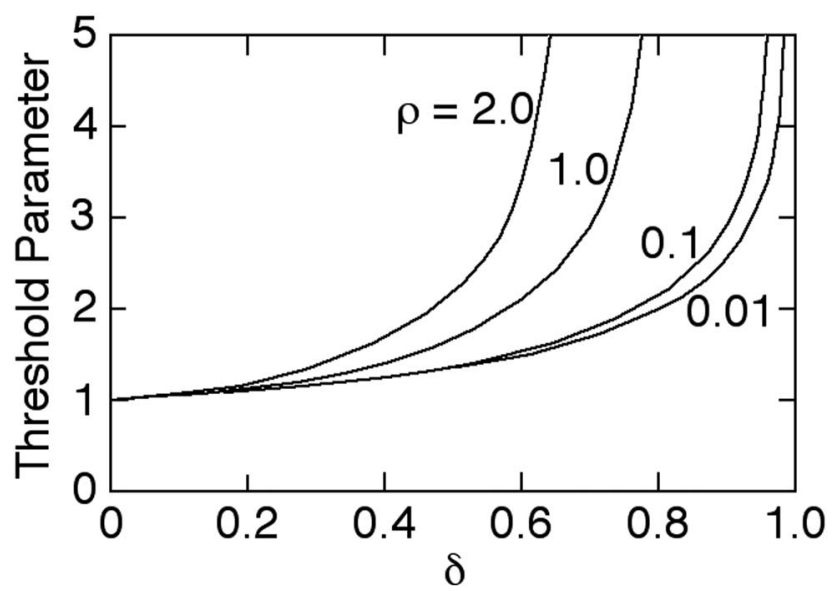

Fig. 1. Type-1 stability boundaries for inhomogeneously broadened unidirectional ring laser oscillators with uniform planewave electric fields. cay rate $\gamma_{c}=5 \times 10^{8} \mathrm{sec}^{-1}$. Therefore the normalized parameters needed to represent the xenon laser in Fig. 1 include $\delta=\gamma / \gamma_{c}=16.3 \times 10^{6} / 5 \times 10^{8}=0.033$ and $\rho=\gamma_{d} / \gamma$ $=8.33 \times 10^{5} / 16.3 \times 10^{6}=0.051$. With these parameters, it follows from the figure that the pulsation threshold is almost indistinguishable from the ordinary lasing threshold $\mathfrak{R}=1$. For near threshold operating conditions the output power level in xenon lasers is in the low microwatt range, but spontaneous pulsations were found to occur from the lowest pump levels at which time domain measurements could be achieved.

\section{DISCUSSION}

Spontaneous pulsations in lasers have been of substantial theoretical and experimental interest for many years. Particular attention has been devoted to cases in which the pulsations occur with no extra nonlinearities supplementing the basic laser equations. In this study stability criteria have been derived for inhomogeneously broadened lasers, and in contrast to previous studies these criteria allow arbitrary values of the decay rates for polarization, population difference, and electric field.

The results described here should be helpful in discovering other lasers that might exhibit the semiclassical pulsation instability. Gas lasers with cw excitation would seem to offer some of the best possibilities for finding such lasers, and it is significant that examples to date include the $3.51 \mu \mathrm{m}$ xenon and the $3.39 \mu \mathrm{m}$ helium-neon lasers. Many gas lasers operate under conditions of inhomogeneous (Doppler) broadening and this type of broadening permits a very low instability threshold. From Fig. 1 it follows that the parameter $\delta=\gamma / \gamma_{c}$ should be significantly less than unity, and this means that the field decay $\gamma_{c}$ should be larger than the polarization decay rate $\gamma$. Low-pressure gas lasers can have long polarization lifetimes, and high-gain gas lasers can have very short field lifetimes. To find examples of spontaneously pulsing lasers, one should be particularly interested in cw gas lasers having high gain. Super-radiance (or superfluorescence/amplified spontaneous emission) is one indicator of high gain; for example, super-radiance is observed in cw krypton at $2.523 \mu \mathrm{m}$ and in $\mathrm{cw}$ xenon at $2.026 \mu \mathrm{m}$ [18].

Besides the general physical interest in spontaneous laser pulsations, there is also the possibility of practical applications. One of the features of the spontaneous pulsations discussed here is the dramatic increase in average power that occurs when the laser is pulsing. For nonlinear applications it is also important to recognize that, under pulsing conditions, this power is condensed into spikes, which could further enhance nonlinear effects [9].

For optical communication applications there may sometimes be efficiency advantages in having the initial carrier source be self-organized to produce a periodic pulse train. Also, the chaotic Lorenz attractor, familiar from theoretical studies of laser instabilities, has been employed in an electronic realization for the coding and decoding of transmitted messages [19]. With wellcharacterized chaotic laser systems, similar information processing might be achievable in purely optical networks. 


\section{REFERENCES}

1. L. W. Casperson, in Laser Physics, J. D. Harvey and D. F Walls, eds., Vol. 182 of Springer Lecture Notes in Physics (Springer-Verlag, 1983), pp. 88-106.

2. L. W. Casperson, "Spontaneous coherent pulsations in laser oscillators," IEEE J. Quantum Electron. QE-14, 756-761 (1978).

3. J. Bentley and N. B. Abraham, "Mode-pulling, modesplitting and pulsing in high-gain lasers," J. Opt. Soc. Am. 70, 1622 (1980).

4. J. Bentley and N. B. Abraham, "Mode-pulling, modesplitting and pulsing in a high gain He-Xe laser," Opt. Commun. 41, 52-56 (1982).

5. M. Maeda and N. B. Abraham, "Measurements of modesplitting self-pulsing in a single-mode, Fabry-Perot laser," Phys. Rev. A 26, 3395-3403 (1982).

6. R. S. Gioggia and N. B. Abraham, "Single-mode self-pulsing instabilities at the Lamb dip of a He-Ne $3.39 \mu \mathrm{m}$ laser," Opt. Commun. 47, 278-282 (1983).

7. R. S. Gioggia and N. B. Abraham, "Anomalous mode pulling, instabilities, and chaos in a single mode, standingwave $3.39 \mu \mathrm{m}$ He-Ne laser," Phys. Rev. A 29, 1304-1309 (1984).

8. P. Chenkosol and L. W. Casperson, "Spontaneous coherent pulsations in $3.39 \mu \mathrm{m}$ He-Ne Standing-Wave Laser Oscillators," J. Opt. Soc. Am. B 20, 2539-2547 (2003).

9. L. W. Casperson, "Spontaneous coherent pulsations in ringlaser oscillators," J. Opt. Soc. Am. B 2, 62-72 (1985).

10. D. K. Bandy, L. M. Narducci, L. A. Lugiato, and N. B.
Abraham, "Time-dependent behavior of a unidirectional ring laser with inhomogeneous broadening," J. Opt. Soc. Am. B 2, 56-61 (1985).

11. L. W. Casperson, "Spontaneous coherent pulsations in standing-wave laser oscillators," J. Opt. Soc. Am. B 5, 958-969 (1988).

12. P. Mandel, "Influence of Lorentz broadening on the stability of monomode ring lasers," Opt. Commun. 45, 269-272 (1983).

13. L. A. Lugiato, L. M. Narducci, D. K. Bandy, and N. B. Abraham, "Instabilities in inhomogeneously broadened single-mode lasers," Opt. Commun. 46, 115-120 (1983).

14. P. Mandel, "Influence of Doppler broadening on the stability of monomode ring lasers," Opt. Commun. 44, 400-404 (1983).

15. L. W. Casperson, "Spontaneous coherent pulsations in ringlaser oscillators: stability criteria," J. Opt. Soc. Am. 2, 993-997 (1985), and references.

16. L. W. Casperson, "Stability criteria for lasers with mixed line broadening," Opt. Quantum Electron. 19, 29-36 (1987)

17. M. L. Minden and L. W. Casperson, "Dispersion-induced instability in cw laser oscillators," IEEE J. Quantum Electron. QE-18, 1952-1957 (1982)

18. C. S. Willett, "Neutral gas lasers," in Handbook of Lasers with Selected Data on Optical Technology, R. J. Pressley, ed. (CRC Press, 1971) pp. 183-241.

19. K. M. Cuomo and A. V. Oppenheim, "Circuit implementation of synchronized chaos with applications to communications,” Phys. Rev. Lett. 71, 65-68 (1993). 